\title{
EFFECTS OF THE POROUS BOUNDARY AND INCLINED MAGNETIC FIELD ON MHD FLOW IN A RECTANGULAR DUCT
}

\author{
Muhim Chutia \\ Department of Mathematics, Mariani College, Mariani-785634, Assam, India \\ muhimchutia@gmail.com
}

Received: 14 September 2020; Accepted: 8 December 2020

\begin{abstract}
In this work, a steady two dimensional MHD flow of a viscous incompressible fluid through a rectangular duct under the action of an inclined magnetic field with a porous boundary has been investigated. The coupled partial differential equations are transformed into a system of algebraic equations using the finite difference method and are then solved simultaneously using the Gauss Seidal iteration method by programming in Matlab software. Numerical solutions for velocity, induced magnetic field and current density lines are obtained and analyzed for different values of dimensionless parameters namely suction/ /injection parameter $(S)$, Hartmann number $(M)$ and inclination angle $(\theta)$ and are presented graphically.
\end{abstract}

MSC 2010: 76D05, 76W05, 76M20

Keywords: $M H D$, rectangular duct, porous boundary, inclined magnetic field, finite difference method

\section{Introduction}

The theoretical or numerical study of magnetohydrodynamic (MHD) flow through ducts has gained great interest to many researchers because of its wide applications in science and engineering such as in the cooling system of nuclear reactors, MHD generators and pumps, blood flow measurement, MHD flow meters and accelerators, etc. Describing equations of MHD flow are the combined and modified equations of the Navier-Stokes equations of fluid mechanics and the Maxwell's equations of electrodynamics. Due to the coupling of equations of these two branches of science, exact solutions are possible only for some simple situations and geometry. So, various numerical techniques are effective for the approximate solutions of MHD flow problems for complex situations and geometry.

Many researchers studied MHD flows through a non-porous rectangular duct in two-dimensional (i.e., the velocity and induced magnetic field depend on two coordinates) cases considering different aspects of the flow problems. Among them, in 1953, Shercliff [1] first obtained an exact solution for two-dimensional MHD flows 
in rectangular ducts with insulating walls in the presence of a transverse magnetic field. He pointed out the existence of a second type of boundary layer known as side or parallel layers near walls parallel to the magnetic field besides the Hartmann layers. Chang and Lundgren [2] and Fendoglu et al. [3] analytically studied MHD flow in a rectangular duct. Celik [4], Kim [5] and Chutia and Deka [6] solved MHD flow equations numerically in rectangular/square ducts for different aspects of the problems. Tayebi and Chamkha [7] numerically studied the steady natural convective heat transfer and flow characteristics of an $\mathrm{Al}_{2} \mathrm{O}_{3}-\mathrm{Cu} /$ water hybrid nanofluid filled square enclosure in the presence of magnetic field equipped with a wavy circular conductive cylinder. Ghalambaz et al. [8] investigated the effects of a hybrid nanofluid in a square cavity that was divided into two equal parts by a vertical flexible partition in the presence of a magnetic field. Chamkha et al. [9] numerically investigated the entropy generation and natural convection inside a $\mathrm{C}$-shaped cavity filled with $\mathrm{CuO}$-water nanofluid and subjected it to a uniform magnetic field by finite volume method with the simple algorithm. Alsabery et al. [10] numerically studied the MHD mixed convection of nanofluid in a lid-driven square cavity subjected to heating by a triangular thick wall. Raza et al. [11] examined the combined effects of thermal radiation and magnetic field of molybdenum disulfide nanofluid in a channel with changing walls with different shapes. Ghalambaz et al. [12] investigated the melting flow and heat transfer of electrically conductive phase change materials subjecting them to a non-uniform magnetic field addressed in a square enclosure. Umavathi and Chamkha [13] numerically investigated the steady natural convection flow for a hydrodynamics case in a vertical rectangular duct employing the finite difference method (FDM).

In all the above studies, the effect of the porous boundary were not considered. It may be well-known that the study of MHD flow through a duct with a porous boundary is significant due to its varied applications in many agricultural and engineering problems such as in designing of cooling systems with liquid metals, nuclear reactors using liquid metal coolant, geothermal energy extraction, underground energy transport, biological and blood flow problems. Veera Krishna and Chamkha [14] discussed the diffusion-thermo, radiation-absorption and Hall and ion slip effects on MHD free convective rotating flow of nanofluids $\left(\mathrm{Ag}\right.$ and $\left.\mathrm{TiO}_{2}\right)$ past a semi-infinite permeable moving plate with constant heat source making use of the perturbation technique. A few works studied by Sai and Nageswara Rao [15], Ramana Murthy et al. [16] and Ramana Murthy and Bahali [17] have been devoted to MHD flow in a rectangular or circular duct with porous walls. Chamkha et al. [18] explored heat transfer and magneto-hydrodynamic flow of hybrid nanofluid in a rotating system among two surfaces assuming the upper and lower plates of the system are penetrable and stretchable respectively, considering thermal radiation and Joule heating impacts.

Perhaps no work has been devoted to MHD flow in a rectangular duct with a porous boundary in the presence of an inclined magnetic field. In this paper, an attempt has been made to numerically study the steady two-dimensional MHD flow of a viscous incompressible electrically conducting fluid through a rectangular 
duct under the action of an inclined magnetic field with porous walls. The problem is described by coupled partial differential equations for velocity and induced magnetic field. Numerical solutions have been obtained for the velocity and the induced magnetic field by developing finite difference codes in Matlab programming that are useful for obtaining current density lines. The solutions for velocity, induced magnetic field and current density lines are analyzed for different values of the suction/injection parameter $(S)$, Hartmann number $(M)$ and inclination angle $(\theta)$ are presented graphically.

\section{Governing equations}

The basic governing equations of MHD flow considered in this problem are the modified Navier-Stokes equation motion (Muller \& Buhler [19]), the magnetic induction equation, Ampere's law and solenoidal properties of velocity and magnetic field:

$$
\begin{gathered}
\rho\left\{\frac{\partial \vec{V}}{\partial t}+(\vec{V} \cdot \nabla) \vec{V}\right\}=-\nabla p+\rho v \nabla^{2} \vec{V}+\vec{J} \times \vec{B} \\
\frac{\partial \vec{B}}{\partial t}=\nabla \times(\vec{V} \times \vec{B})+\lambda \nabla^{2} \vec{B} \\
\nabla \times \vec{B}=\mu_{e} \vec{J} \\
\nabla \cdot \vec{V}=0 \\
\nabla \cdot \vec{B}=0
\end{gathered}
$$

where $\rho, p, v, \mu_{e}, \sigma, \vec{V}, \vec{B}$ and $\vec{J}$ are fluid density, pressure, kinematic viscosity, magnetic permeability, electrical conductivity, velocity vector, magnetic field vector and current density vector respectively.

\section{Mathematical formulation}

We consider the steady two-dimensional laminar flow of a viscous incompressible electrically conducting fluid in a porous rectangular duct under the action of an external uniform applied magnetic field of strength $B_{0}$, which makes an angle $\theta$ with the positive direction of the $y^{\prime}$-axis (i.e., with the positive direction of the non-porous walls) and induces a magnetic field of strength $B_{z^{\prime}}\left(x^{\prime}, y^{\prime}\right)$ in the flow direction. The flow is driven by a constant pressure gradient $\frac{\partial p}{\partial z^{\prime}}$. It is assumed that the walls of the duct are electrically non-conducting. The non-porous walls of the duct are situated at $x^{\prime}= \pm a$, and porous walls are situated at $y^{\prime}= \pm b$ as shown in Figure 1. The axis of the duct is chosen as the $z^{\prime}$-axis. The fluid particles are sucked and injected at the pair of walls parallel to $x^{\prime}$-axis i.e., the fluid particles are 
sucked with a constant velocity $V_{0}$ at $y^{\prime}=-b$ and injected with same velocity at $y^{\prime}=b$. All physical quantities except the pressure gradient are assumed to be independent of variable $z^{\prime}$. Under these assumptions the velocity and magnetic field take the forms:

$$
\vec{V}=\left\{0, V_{0}, V_{z^{\prime}}\left(x^{\prime}, y^{\prime}\right)\right\} \text { and } \vec{B}=\left\{B_{0} \sin \theta, B_{0} \cos \theta, B_{z^{\prime}}\left(x^{\prime}, y^{\prime}\right)\right\}
$$

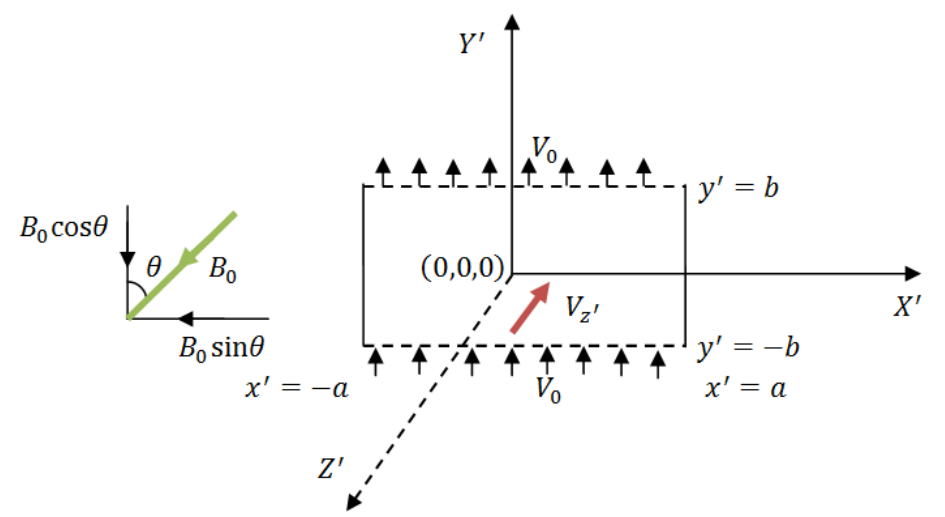

Fig. 1. A cross-section of the rectangular duct normal to the flow direction $\left(z^{\prime}\right.$-axis $)$

In the steady case and using solenoidal properties (4) and (5), Eqs. (1) and (2) can be written as

$$
\begin{gathered}
\rho(\vec{V} \cdot \nabla) \vec{V}=-\nabla p+\rho v \nabla^{2} \vec{V}+\vec{J} \times \vec{B} \\
(\vec{V} \cdot \nabla) \vec{B}=(\vec{B} \cdot \nabla) \vec{V}+\lambda \nabla^{2} \vec{B}
\end{gathered}
$$

Substituting the expressions for the velocity and magnetic field as stated above in Eqs. (6) and (7), we obtain

$$
\begin{gathered}
\rho V_{0} \frac{\partial V_{z^{\prime}}}{\partial y^{\prime}}=-\frac{\partial p}{\partial z^{\prime}}+\frac{B_{0} \sin \theta}{\mu_{e}} \frac{\partial B_{z^{\prime}}}{\partial x^{\prime}}+\frac{B_{0} \cos \theta}{\mu_{e}} \frac{\partial B_{z^{\prime}}}{\partial y^{\prime}}+\rho v\left(\frac{\partial^{2} V_{z^{\prime}}}{\partial x^{\prime 2}}+\frac{\partial^{2} V_{z^{\prime}}}{\partial y^{\prime 2}}\right) \\
V_{0} \frac{\partial B_{z^{\prime}}}{\partial y^{\prime}}=\frac{B_{0} \sin \theta}{\mu_{e}} \frac{\partial V_{z^{\prime}}}{\partial x^{\prime}}+\frac{B_{0} \cos \theta}{\mu_{e}} \frac{\partial V_{z^{\prime}}}{\partial y^{\prime}}+\lambda\left(\frac{\partial^{2} B_{z^{\prime}}}{\partial x^{\prime 2}}+\frac{\partial^{2} B_{z^{\prime}}}{\partial y^{\prime 2}}\right)
\end{gathered}
$$

The Eqs. (8) and (9) are to be solved subject to the following boundary conditions:

$$
\left.\begin{array}{c}
V_{z^{\prime}}=0, B_{z^{\prime}}=0 \text { at } x^{\prime}=-a \\
V_{z^{\prime}}=0, B_{z^{\prime}}=0 \text { at } x^{\prime}=a \\
V_{z^{\prime}}=0, B_{z^{\prime}}=0 \text { at } y^{\prime}=-b \\
V_{z^{\prime}}=0, B_{z^{\prime}}=0 \text { at } y^{\prime}=b
\end{array}\right\}
$$

In this problem, we define the following dimensionless quantities: 


$$
x=\frac{x^{\prime}}{a}, y=\frac{y^{\prime}}{A a}, V=\frac{V_{z^{\prime}}}{V_{0}}, B=\frac{B_{z^{\prime}}}{B_{0}}
$$

where: $V_{0}=-\frac{a^{2}}{\rho v} \frac{\partial p}{\partial z^{\prime}}$ and $B_{0}=-a^{2} \mu_{e}\left(\frac{\sigma}{\rho v}\right)^{1 / 2} \frac{\partial p}{\partial z^{\prime}}$.

Using dimensionless quantities (11) in Eqs. (8) and (9), we obtain

$$
\begin{aligned}
& S \frac{\partial V}{\partial y}=1+M \sin \theta \frac{\partial B}{\partial x}+\frac{1}{A} M \cos \theta \frac{\partial B}{\partial y}+\frac{\partial^{2} V}{\partial x^{2}}+\frac{1}{A^{2}} \frac{\partial^{2} V}{\partial y^{2}} \\
& S P r_{m} \frac{\partial B}{\partial y}=M \sin \theta \frac{\partial V}{\partial x}+\frac{1}{A} M \cos \theta \frac{\partial V}{\partial y}+\frac{\partial^{2} B}{\partial x^{2}}+\frac{1}{A^{2}} \frac{\partial^{2} B}{\partial y^{2}}
\end{aligned}
$$

where $S=\frac{V_{0} a}{v}$ is the suction/injection parameter, $P r_{m}=\sigma \mu_{e} v$ is the magnetic Prandtl number, $M=B_{0} a\left(\frac{\sigma}{\rho v}\right)^{1 / 2}$ is the Hartmann number and $A=\frac{b}{a}$ is the aspect ratio.

Then the boundary conditions (10) in dimensionless forms can be rewritten as follows:

$$
\left.\begin{array}{l}
V=0, B=0 \text { at } x=-1 \\
V=0, B=0 \text { at } x=1 \\
V=0, B=0 \text { at } y=-1 \\
V=0, B=0 \text { at } y=A
\end{array}\right\}
$$

\section{Numerical solution}

The dimensionless coupled linear partial differential equations (12) and (13) with the prescribed boundary conditions (14) are simplified employing the finite difference scheme to obtain a system of algebraic equations. Central difference approximation is used to discretize the coupled PDEs, since it is more accurate than the forward and backward differences. So, both the first and second derivative terms appearing in the coupled equations are descretized employing the central difference scheme of second order accuracy. In the finite difference techniques, we have to divide the computational domain into a uniform grid system. We divide the rectangular region bounded by $-1 \leq x \leq 1$ and $-1 \leq y \leq A$ into a grid consisting of $(m+1)$ by $(n+1)$ rectangle with sides $\Delta x=h$ and $\Delta y=k$. The grid points are identified by two subscripts $i: i=1,2,3, \ldots,(m+1)$ and $j: j=1,2,3$, $\ldots,(n+1)$. Where index $i$ refers to $x$ and $j$ refers to $y$. Numerical meshes $-1=$ $=x_{1}<x_{2}<x_{3} \ldots<x_{m+1}=1$ and $-1=y_{1}<y_{2}<y_{3} \ldots<y_{n+1}=A$ are considered along $x$ and $y$-axes. Central difference approximations for both first and second derivatives of second order accuracy of the equations (12) and (13) are as follows: 


$$
\begin{aligned}
S\left(\frac{V_{i, j+1}-V_{i, j-1}}{2 k}\right)= & 1+M \sin \theta\left(\frac{B_{i+1, j}-B_{i-1, j}}{2 h}\right)+\frac{1}{A} M \cos \theta\left(\frac{B_{i, j+1}-B_{i, j-1}}{2 k}\right) \\
+ & \frac{V_{i+1, j}-2 V_{i, j}+V_{i-1, j}}{h^{2}}+\frac{1}{A^{2}}\left(\frac{V_{i, j+1}-2 V_{i, j}+V_{i, j-1}}{k^{2}}\right) \\
\operatorname{SPr}_{m}\left(\frac{B_{i, j+1}-B_{i, j-1}}{2 k}\right) & =M \sin \theta\left(\frac{V_{i+1, j}-V_{i-1, j}}{2 h}\right)+\frac{1}{A} M \cos \theta\left(\frac{V_{i, j+1}-V_{i, j-1}}{2 k}\right) \\
& +\frac{B_{i+1, j}-2 B_{i, j}+B_{i-1, j}}{h^{2}}+\frac{1}{A^{2}}\left(\frac{B_{i, j+1}-2 B_{i, j}+B_{i, j-1}}{k^{2}}\right)
\end{aligned}
$$

The corresponding discretized boundary conditions are as follows:

$$
\begin{aligned}
& V_{1, j}=0, \quad B_{1, j}=0 \quad \text { at } i=1 \\
& V_{m+1, j}=0, B_{m+1, j}=0 \text { at } i=m+1 \\
& V_{i, 1}=0, \quad B_{i, 1}=0 \quad \text { at } j=1 \\
& V_{i, n+1}=0, \quad B_{i, n+1}=0 \text { at } j=n+1
\end{aligned}
$$

Equations (15) and (16) separately represents a system of linear $(m-1)(n-1)$ equations in $(m-1)(n-1)$ unknown values for $V$ and $B$. Using initial and boundary conditions (17), and selecting the dimensionless parameters involved in dimensionless equations, the system linear equations are solved simultaneously by the Gauss Seidal iteration method, developing finite difference codes in Matlab programming (Mathews \& Fink [20]). Thus $V$ and $B$ are known at all values of $x$ and $y$. The convergences of each of the computed values of variables $V$ and $B$ at different grid points are checked by Root-Mean-Square residuals $R_{S}$ (Al-khawaja and Selmi [21]). Convergence can be considered to be achieved, where $R_{S}<10^{-7}$; where

$$
\begin{aligned}
& R_{V}=\sqrt{\sum_{i=2}^{m} \sum_{j=2}^{n}\left(V_{i, j}^{N+1}-V_{i, j}^{N}\right)^{2}} \\
& R_{B}=\sqrt{\sum_{i=2}^{m} \sum_{j=2}^{n}\left(B_{i, j}^{N+1}-B_{i, j}^{N}\right)^{2}}
\end{aligned}
$$

\section{Results and discussion}

In this paper, steady two dimensional MHD flow of a viscous incompressible electrically conducting fluid through a rectangular duct in the presence of an inclined magnetic field with porous boundary have been investigated. The pairs of porous and non-porous walls are assumed to be non-conducting and the applied magnetic field makes an angle with non-porous walls. The problem is described by the coupled partial differential equation for velocity and the induced magnetic 
field. Numerical solutions for the velocity, induced magnetic field and current density lines have been obtained by developing finite difference codes in Matlab programming with the help of Software Package "MATLAB R2008b". In all these computations, $A=1$ and $P r_{m}=1$ are fixed to discuss the effects of other nondimensional flow parameters and a uniform $101 \times 101$ mesh size is used.

Figures 2a-e show the effect of inclination angle $\theta$ on 3D velocity distributions $V$ for $M=100$ and $S=15$. It is observed in these figures that the fluid velocity increases and rotates as the inclination angle $\theta$ of the applied magnetic field increases. Since the applied magnetic field makes an angle $\theta$ with the $y$-axis, and the strength of the applied magnetic field decreases for increasing values of inclination angles.

The effects of inclination angle $\theta$ on the 3D induced magnetic field are presented in Figures $2 \mathrm{f}-\mathrm{j}$ for $M=100$ and $S=15$. It is also seen that the induced magnetic field increases and rotates as the inclination angle $\theta$ increases.

Figures $2 \mathrm{k}$-o present the effect of inclination angle $\theta$ on $2 \mathrm{D}$ current density lines $\left[J_{x}, J_{y}\right]$ for $M=100$ and $S=15$. We have noticed in these figures that the current density lines rotate and the rotation increases as the inclination angle $\theta$ increases. It is also observed that the Hartmann layers are formed perpendicular to the direction of applied magnetic field, and side layers are formed parallel to the direction of applied magnetic field. These well known MHD phenomena are seen in these figures clearly.

Figures 3a-c depict the effect of Hartmann $M$ on 3D velocity distribution respectively for $M=50,100$ and 150 ; it is noticed that the velocity distribution decreases for the increasing values of the Hartmann number.

The effects of Hartmann number $M$ on the 3D induced magnetic field are presented in Figures 4a-c respectively for $M=50,100$ and 150; it is seen that induced magnetic field decreases as the Hartmann number $M$ increases.

Figures 5a-c present the effect of Hartmann number $M$ on 2D current density lines $\left[J_{x}, J_{y}\right]$ respectively for $M=50,100$ and 150 . It is observed that the current density lines become flattened and segregated to a narrow region normal to the direction of the applied magnetic field called Hartmann layers as the Hartmann number $M$ increases.

In Figures 6a-c, we have presented the effect of suction parameter $S(S>0)$ on $3 \mathrm{D}$ velocity distribution respectively for $S=5,15$ and 25; it is noticed that the fluid velocity decreases near the sucked wall and increases near the injected wall due to increasing values of suction parameter. Reverse effects are seen in Figures $7 \mathrm{a}-\mathrm{c}$ for increasing values of injection parameter $(S<0)$.

The effect of suction parameter $S(S>0)$ on the 3D induced magnetic field are presented in Figures 8a-c respectively for $S=5,15$ and 25; it is seen that induced magnetic decreases near the sucked wall and increases near the injected wall as the suction parameter $S$ increases. Reverse effects are seen in Figures 9a-c as the injection parameter $S(S<0)$ increases. 


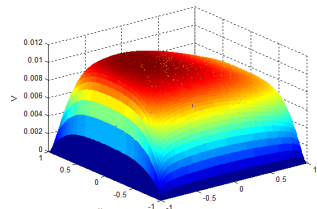

(a) $\theta=0^{\circ}$

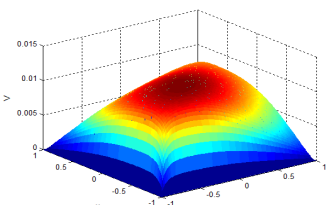

(b) $\theta=30^{\circ}$

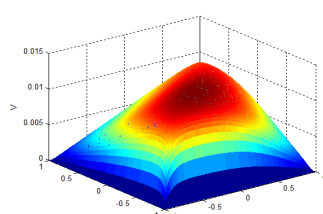

(c) $\theta=45^{\circ}$

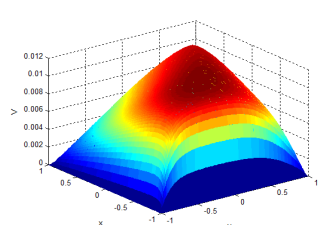

(d) $\theta=60^{\circ}$

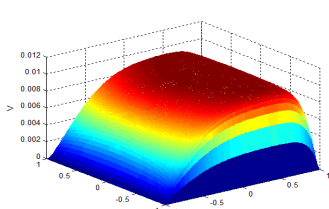

(e) $\theta=90^{\circ}$

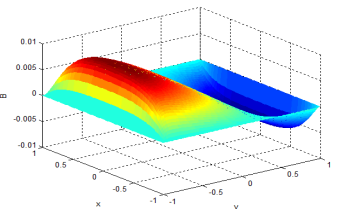

(f) $\theta=0^{\circ}$

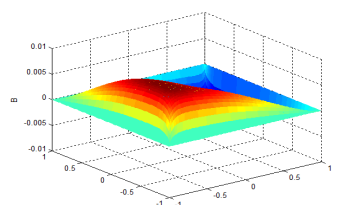

(g) $\theta=30^{\circ}$

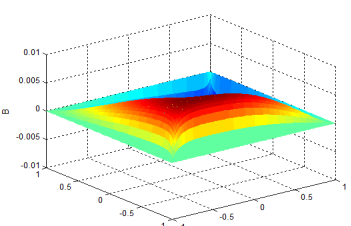

(h) $\theta=45^{\circ}$

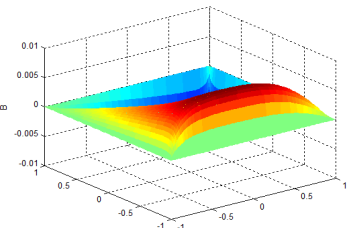

(i) $\theta=60^{\circ}$

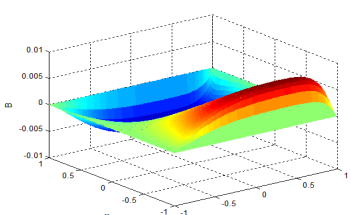

(j) $\theta=90^{\circ}$

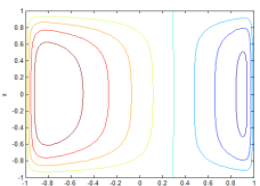

(k) $\theta=0^{\circ}$

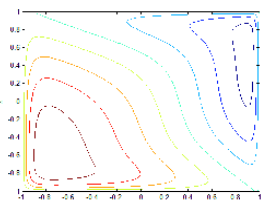

(1) $\theta=30^{\circ}$

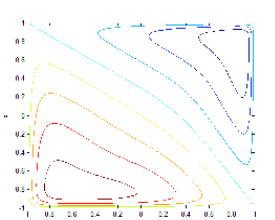

(m) $\theta=45^{\circ}$

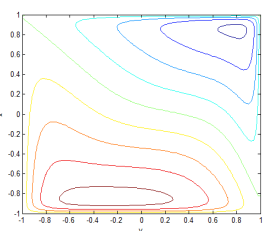

(n) $\theta=60^{\circ}$

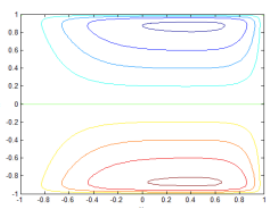

(o) $\theta=90^{\circ}$

Fig. 2. 3D velocity (a-e); induced magnetic field (f-j); $2 \mathrm{D}$ current density lines (k-o) at various inclination angle $\theta$ for $S=15$ and $M=50$

Figures 10a-c depict that current density lines $\left[J_{x}, J_{y}\right]$ close its paths in boundary layers and swell towards the sucked wall and compress towards the injected wall as the suction parameter $S(S>0)$ increases. Reverse effects are observed in Figures $11 \mathrm{a}-\mathrm{c}$ for the increasing values of injection parameter $S(S<0)$. This effect is more relevant for the high suction/injection parameter.

Axial velocity and induced magnetic field for different values inclination angle $\theta$ are presented in Figures 12a-b. It is noticed in these figures that both velocity and induced magnetic field increases as the inclination angle increases. 
Effects of the porous boundary and inclined magnetic field on MHD flow in a rectangular duct

41

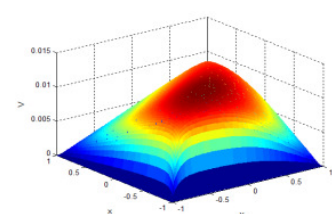

(a) $M=50$

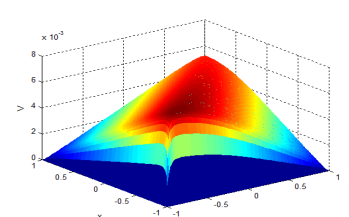

(b) $M=100$

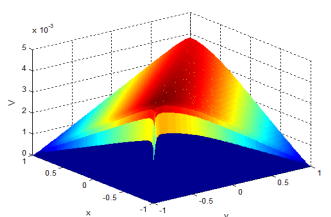

(c) $M=150$

Fig. 3. 3D velocity for $\theta=45^{\circ}$ and $S=15$ at (a) $M=50$; (b) $M=100$; (c) $M=150$

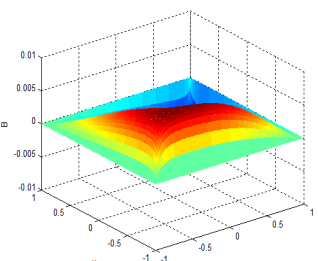

(a) $M=50$

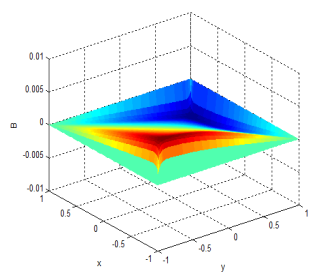

(b) $M=100$

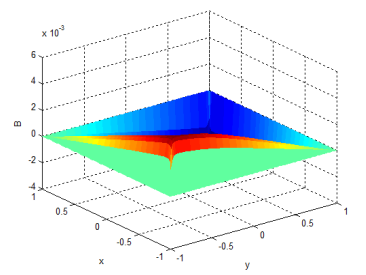

(c) $M=150$

Fig. 4. 3D induced magnetic field for $\theta=45^{\circ}$ and $S=15$ at (a) $M=50$; (b) $M=100$;

(c) $M=150$

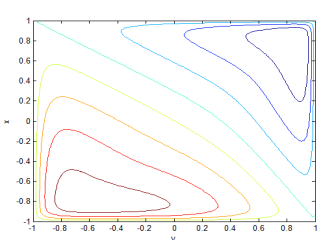

(a) $M \stackrel{y}{=} 50$

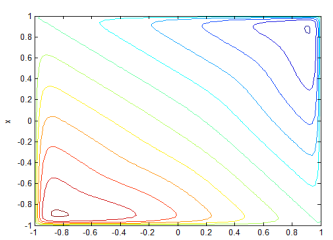

(b) $M=100$

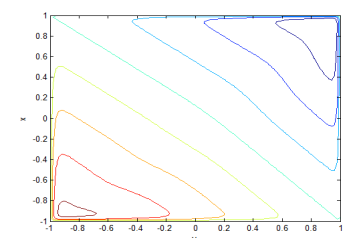

(c) $M=150$

Fig. 5. Current density lines $\left[J_{x}, J_{y}\right]$ for $\theta=45^{\circ}$ and $S=15$ at (a) $M=50$; (b) $M=100$;

(c) $M=150$

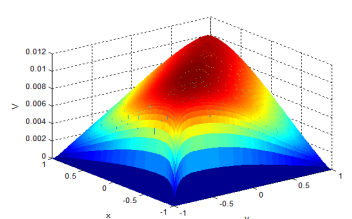

(a) $S=5$

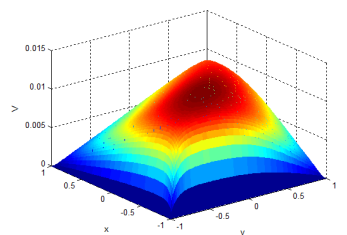

(b) $S=15$

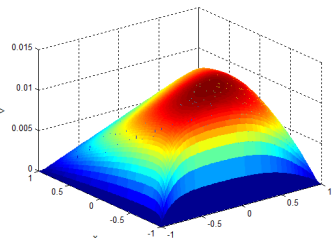

(c) $S=25$

Fig. 6. $3 \mathrm{D}$ velocity for $\theta=45^{\circ}$ and $M=50$ at (a) $S=5$; (b) $S=15$; (c) $S=25$

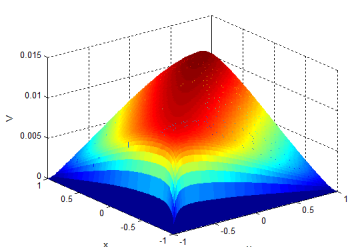

(a) $S=-5$

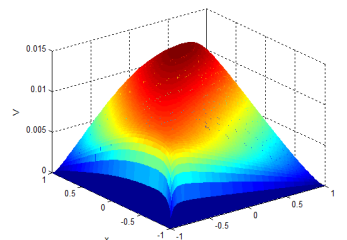

(b) $S=-15$

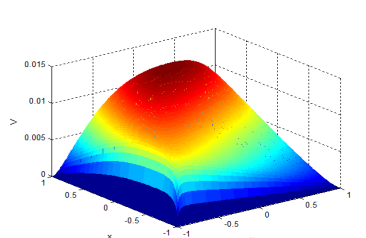

(c) $S=-25$

Fig. 7. 3D velocity for $\theta=45^{\circ}$ and $M=50$ at (a) $S=-5$; (b) $S=-15$; (c) $S=-25$ 


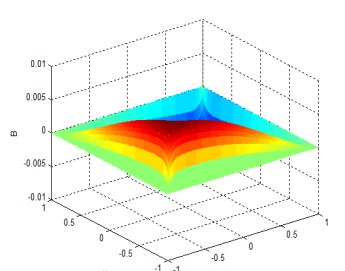

(a) $S=5$

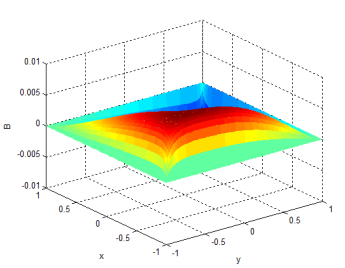

(b) $S=15$

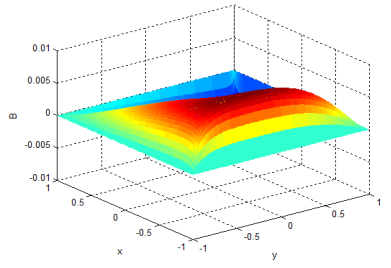

(c) $S=25$

Fig. 8. 3D induced magnetic field for $\theta=45^{\circ}$ and $M=50$ at (a) $S=5$; (b) $S=15$;

(c) $S=25$

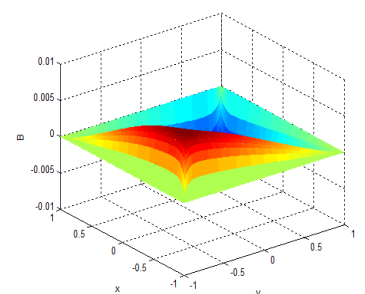

(a) $S=-5$

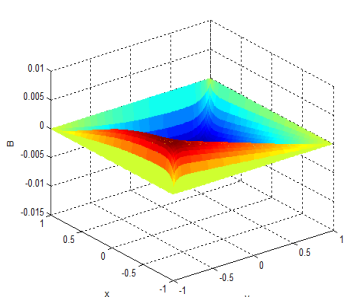

(b) $S=-15$

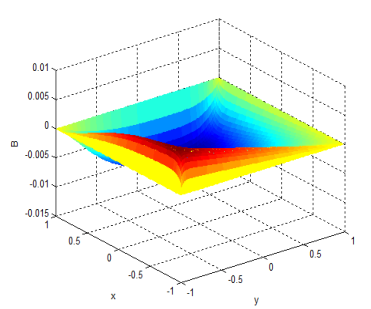

(c) $S=-25$

Fig. 9. 3D induced magnetic field for $\theta=45^{\circ}$ and $M=50$ at (a) $S=-5$; (b) $S=-15$;

(c) $S=-25$

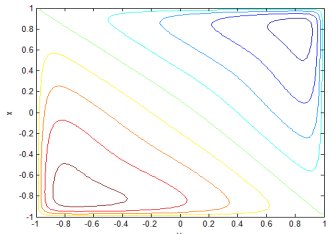

(a) $S=5$

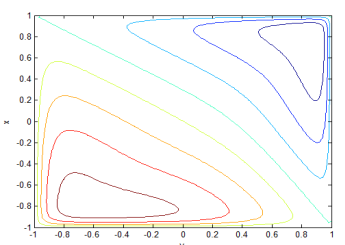

(b) $S=15$

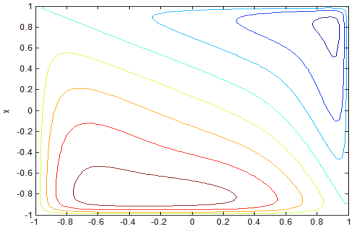

(c) $S=25$

Fig. 10. Current density lines $\left[J_{x}, J_{y}\right]$ for $\theta=45^{\circ}$ and $M=50$ at (a) $S=5$; (b) $S=15$;

(c) $S=25$

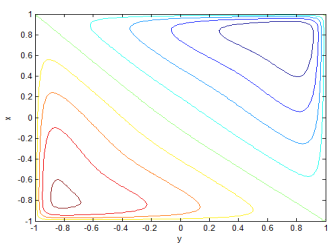

(a) $S=-5$

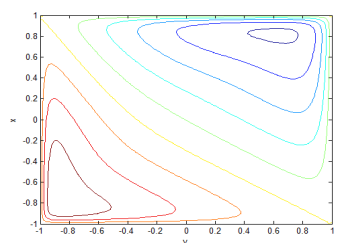

(b) $S=-15$

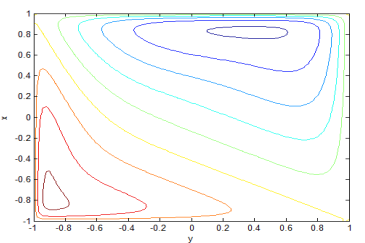

(c) $S=-25$

Fig. 11. Current density lines $\left[J_{x}, J_{y}\right]$ for $\theta=45^{\circ}$ and $M=50$ at (a) $S=-5$; (b) $S=-15$;

(c) $S=-25$ 


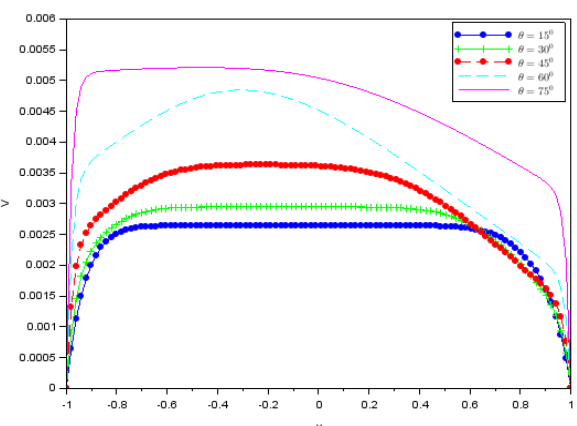

(a) $V$ at various $\theta$

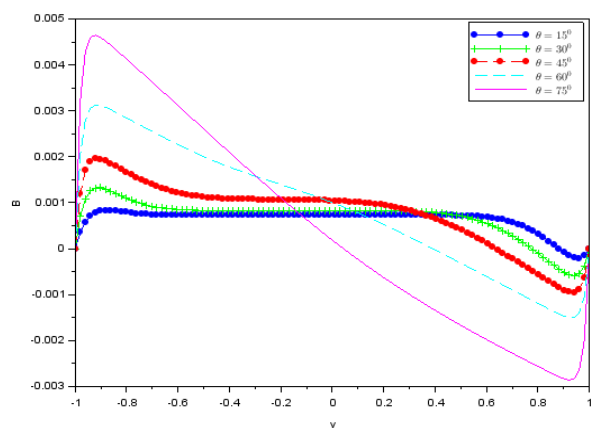

(b) $B$ at various $\theta$

Fig. 12. Axial velocity (a) and induced magnetic field (b) at various $\theta$ for $M=100$ and $S=5$

\section{Conclusions}

In this paper, the effects of porous boundary and inclined magnetic fields on steady two-dimensional flow of an electrically conducting viscous fluid in a rectangular duct have been investigated. The dimensionless partial differential equations of momentum and induction are transformed into simpler algebraic equations using finite difference schemes which are then solved numerically by developing codes in Matlab programming. Computed results presented above in terms of graphics are listed below:

1. Both velocity and induced magnetic field increases and rotates as the inclination angle $\theta$ of the applied magnetic field increases.

2. Current density lines rotate, and rotation increases as the inclination angle $\theta$ increases. Hartmann layers are formed perpendicular to the direction of the applied magnetic field and side layers are formed parallel to the direction of the applied magnetic field.

3. Both velocity and induced magnetic field decreases and become flattened as the Hartmann number $M$ increases.

4. Current density lines become flattened and segregated to a narrow region normal to the direction of the applied magnetic field called Hartmann layers, as the Hartmann number $M$ increases.

5. Both velocity and induced magnetic field decreases near the sucked wall and increases near the injected wall for increasing values of suction parameter $S(S>0)$. Reverse effects occur for increasing values of injection parameter $S(S<0)$.

6. Current density lines close their paths in boundary layers and swell towards the sucked wall and compress towards the injected wall as the suction parameter $S$ $(S>0)$ increases. Reverse effects occur when injection parameter $S(S<0)$ increases.

\section{References}

[1] Shercliff, J.A. (1953). Steady motion of conducting fluids in pipes under transverse magnetic fields. Mathematical Proceedings of the Cambridge Philosophical Society, 49(1), 136-144. 
[2] Chang, C., \& Lundgren, S. (1961). Duct flow in magnetohydrodynamics. Zeitschrift fur angewandte Mathematik und Physik (ZAMP), 12(2), 100-114.

[3] Fendoglu, H., Bozkaya, C., \& Tezer-Sezgin, M. (2019). MHD flow in a rectangular duct with a perturbed boundary. Computers \& Mathematics with Applications, 77(2), 374-388.

[4] Celik, I. (2011). Solution of magnetohydrodynamics flow in a rectangular duct by Chebyshev collocation method. International Journal for Numerical Methods in Fluids, 66, 1325-1340.

[5] Kim, C.N. (2018). Numerical analysis of a magnetohydrodynamic duct flow with flow channel insert under a non-uniform magnetic field. Journal of Hydrodynamics, 30, 1134-1142.

[6] Chutia, M., \& Deka, P.N. (2015). Numerical solution for coupled MHD flow equations in a square duct in the presence of strong inclined magnetic field. International Journal of Advanced Research in Physical Science, 2(9), 20-29.

[7] Tayebi, T., \& Chamkha, Ali J. (2020). Magnetohydrodynamic natural convection heat transfer of hybrid nanofluid in a square enclosure in the presence of a wavy circular conductive cylinder. Journal of Thermal Science and Engineering Applications, 12(3), 031009.

[8] Ghalambaz, M., Mehryan, S.A.M., Izadpanahi, E., Chamkha, Ali J., \& Wen, D. (2019). MHD natural convection of $\mathrm{Cu}-\mathrm{Al}_{2} \mathrm{O}_{3}$ water hybrid nanofluids in a cavity equally divided into two parts by a vertical flexible partition membrane. Journal of Thermal Analysis and Calorimetry, 138, 1723-1743.

[9] Chamkha, Ali J., Ismael, M., Kasaeipoor, A., \& Armaghani. T. (2016). Entropy generation and natural convection of $\mathrm{CuO}$-water nanofluid in $\mathrm{C}$-shaped cavity under magnetic field. Entropy, 18,50 .

[10] Alsabery, A.I., Armaghani, T., Chamkha, Ali J., \& Hashim, I. (2020). Two-phase nanofluid model and magnetic field effects on mixed convection in a lid-driven cavity containing heated triangular wall. Alexandria Engineering Journal, 59, 129-148.

[11] Raza, J., Mebarek-Oudina, F., \& Chamkha, Ali J. (2019). Magnetohydrodynamic flow of molybdenum disulfide nanofluid in a channel with shape effects. Multidiscipline Modeling in Materials and Structures, 15(4), 737-757.

[12] Ghalambaz, M., Hashem Zadeh, S.M., Mehryan, S.A.M., Pop, I., \& Wen, D. (2020). Analysis of melting behavior of PCMs in a cavity subject to a non-uniform magnetic field using a moving grid technique. Applied Mathematical Modelling, 77(2), 1936-1953.

[13] Umavathi, J.C., \& Chamkha, Ali J. (2013). Steady natural convection flow in a vertical rectangular duct with isothermal wall boundary conditions. International Journal of Energy and Technology, 5, 1-10.

[14] Veera Krishnaa, M., \& Chamkha, Ali J. (2019). Hall and ion slip effects on MHD rotating boundary layer flow of nanofluid past an infinite vertical plate embedded in a porous medium. Results in Physics, 15, 102652.

[15] Sai, K.S., \& Nageswara Rao, B. (2000). Magnetohydrodynamic flow in a rectangular duct with suction and injection. Acta Mechanica, 140, 57-64.

[16] Ramana Murthy, J.V., Sai, K.S., \& Bahali, N.K. (2011). Steady flow of micropolar fluid in a rectangular channel under transverse magnetic field with suction. AIP Advances, 1, 032123-1032123-10.

[17] Raman Murthy, J.V. \& Bahali, N.K. (2009). Steady flow of micropolar fluid through a circular pipe under a transverse magnetic field with constant suction/injection. International Journal of Applied Mathematics and Mechanics, 5(3), 1-10.

[18] Chamkha, Ali J., Dogonchi, A.S. \& Ganji, D.D. (2019). Magneto-hydrodynamic flow and heat transfer of a hybrid nanofluid in a rotating system among two surfaces in the presence of thermal radiation and Joule heating. AIP Advances, 9, 025103.

[19] Muller, U., \& Buhler, L. (2001). Magnetofluiddynamics in Channels and Containers. Springer.

[20] Mathews, J.H., \& Fink, F.D. (2009). Numerical Method using Matlab. New Delhi, PHI Learning Private Limited.

[21] Al-khawaja, M.J., \& Selmi, M. (2010). Matlab Modelling Programming and Simulations. Sciyo, 365-388. 